\author{
David Garibay \\ Maître de conférences en science politique \\ Université Lumière Lyon 2, Laboratoire Triangle (CNRS UMR 5206) \\ Article paru dans \\ Sciences humaines, Les grands dossiers, 6, mars 2007, p. 54-59 \\ Version définitive disponible sur \\ http://www.scienceshumaines.com/comment-l-amerique-latine-bascule-a- \\ gauche_fr_15399.html
}

\title{
Comment l'Amérique latine bascule à gauche
}

Le panorama politique de l'Amérique latine, dans les années 1970, est des plus sombres. Tous les pays d'Amérique du sud, à l'exception de la Colombie et du Venezuela, sont gouvernés par des dictatures militaires. L'Amérique centrale est ravagée par des guerres civiles, nourries par le contexte de la guerre froide. Gouverné par des civils, le Mexique semble faire exception, mais la stabilité repose sur un régime de parti unique qui contrôle les élections, et ne laisse qu'une portion congrue à l'opposition.

\section{Des juntes aux démocraties pérennes}

Pourtant cette situation change rapidement : en une dizaine d'année, tous les régimes militaires d'Amérique du sud disparaissent. Partout des élections libres et transparentes sont organisées. Même les militaires argentin et chilien, qui ont pratiqué la violence d'Etat et la répression de la manière la plus forte et la plus systématique, se retirent dans leurs casernes, respectivement en 1983 pour la junte argentine et en 1989 pour le régime de Pinochet. Cette vague de démocratisation concerne également l'Amérique centrale, où entre 1990 et 1996 des accords négociés permettent en même temps la paix, la démocratie et la participation aux élections des anciens acteurs armés. Au Mexique, le parti unique cède à son tour son monopole sur le pouvoir, d'abord à l'occasion d'élections locales, puis d'élections législatives, et enfin au niveau central lors de l'alternance présidentielle de 2000.

La mutation institutionnelle de l'Amérique latine est d'autant plus remarquable qu'elle est rapide, et qu'elle se produit dans une conjoncture dans laquelle les militaires au pouvoir étaient dans une situation de force -seuls les militaires argentins battus lors de la guerre des Malouines en 1983 par le Royaume-Uni sont véritablement affaiblis. Partout ailleurs, des négociations entre les militaires et les dirigeants de l'opposition commencent plutôt sur une volonté de libéralisation limitée des premiers, et partout ces négociations aboutissent progressivement à une démocratisation effective. La manière dont se réalise la démocratisation conduit néanmoins à une limite importante de ce changement : les militaires demeurant au pouvoir pendant les négociations, ils sont en mesure d'imposer leurs conditions sur un certain nombre de points. Ils excluent ainsi les acteurs les plus radicaux de l'opposition des négociations. Mais surtout ils cherchent à garantir leur survie, en imposant le fait qu'il ne sera pas possible de poursuivre et de condamner des militaires pour des actions commises pendant les périodes dictatoriales. Hormis, une fois encore, le cas argentin, les procédures judiciaires contre des militaires pour violations des Droits de l'homme sont de fait impossibles.

Crise économique, thérapie néolibérale 
Le cas chilien est le plus connu : les militaires imposent une amnistie non négociable, mais s'assurent aussi que le général Pinochet demeure chef de l'Etat-major, que l'armée maintienne une certaine autonomie, que $20 \%$ des sénateurs soient nommés à vie, et qu'enfin le mode de scrutin adopté permette une sur-représentation des partis de droite héritiers de la dictature $^{1}$

Mais, une fois les premières élections convoquées, la nature démocratique du régime n'a pratiquement jamais été remise en question, ni par les militaires ou les anciens dictateurs, ni par l'opposition la plus radicale. A l'exception du Pérou, où Alberto Fujimori utilise son succès dans la lutte armée contre la guérilla maoïste du Sentier lumineux pour renforcer son pouvoir, interrompre en 1992 l'activité du Parlement et adopter une Constitution autoritaire. Réélu en 1995, puis à nouveau en 2000, A. Fujimori doit néanmoins quitter le pouvoir la même année à la suite de mobilisations de rue.

Pourtant si ces transitions sont extrêmement significatives d'un point de vue politique, elles se réalisent dans une conjoncture qui en altère la portée sinon l'importance ${ }^{2}$. En effet les années 1980 sont également celles d'une crise économique généralisée à l'ensemble de la région, où l'explosion de la dette extérieure, l'hyperinflation et la récession voient leurs effets se cumuler. La réponse apportée à cette situation est l'adoption de thérapies de choc néolibérales par les nouveaux gouvernements parvenus au pouvoir lors des premières élections. Suivant les principes du Consensus de Washington en vogue alors dans les institutions multilatérales, le Fonds monétaire international et la Banque mondiale conditionnent leur aides financières à la mise en place de plans d'ajustement structurels. Ceux-ci se traduisent par une rigueur budgétaire, la réduction massive des dépenses publiques, des dérégulations, l'ouverture des marchés financiers, l'ouverture des frontières au commerce international, des privatisations massives et la quasi-élimination des protections accordées aux salariés par le droit du travail et les conventions collectives ${ }^{3}$.

\section{Les conséquences sociales et politiques}

Les recettes appliquées permettent de réduire relativement vite l'inflation, de restaurer certains équilibres macro-économiques et de relancer une certaine croissance économique qui repose néanmoins sur des bases extrêmement fragiles. Mais les effets sociaux de cette politique sont dramatiques, en particulier pour les populations les plus pauvres et pour les classes moyennes. Elles ne sont partiellement compensées que par l'explosion de l'économie informelle et de la migration clandestine vers les Etats-Unis. De ce fait, pour les sociétés latino-américaines, la démocratie se généralise dans un contexte de crise économique sévère et d'approfondissement des inégalités sociales.

D’une certaine manière, la survie des régimes démocratiques dans un contexte socioéconomique aussi difficile est une preuve tangible de la solidité des nouvelles institutions. Mais ta mise en oeuvre de réformes néolibérales signifie la réduction de trois caractéristiques centrales assurées par l'Etat et plus largement par le politique : un rôle d'orientation de l'activité économique, un rôle de garant d'une forme de redistribution sociale, enfin une idée du politique dans son ensemble comme lieu de la confrontation d'alternatives.

La réduction du rôle de l'Etat est d'abord une réalité économique (privatisations des entreprises nationales et des services publics, licenciements massifs dans la fonction

\footnotetext{
${ }^{1}$ Le sociologue chilien Manuel Antonio Garretón a qualifié ces conditions d' « enclaves autoritaires ». Pour une évolution de cette situation depuis lors, voir le dossier « Chili : la fin des enclaves autoritaires », Problèmes d'Amérique latine, 56, printemps 2005.

${ }^{2}$ Pour une analyse d'ensemble de la situation contemporaine de la région, voir le dossier « Amérique latine », Questions internationales, 18, mars 2006.

${ }^{3}$ Yves DEZALAY, Bryant GARTH, La mondialisation des guerres de palais, Paris, le Seuil, 2002.
} 
publique), ce qui a des conséquences très significatives dans des pays dans lesquels il existait une certaine tradition d'interventionnisme étatique.

Cette réduction a également une dimension sociale très importante. Les institutions publiques ne se donnent plus en effet comme fonction de garantir une certaine forme de redistribution. Il est vrai que seuls les pays du cône sud (Argentine, Uruguay, Chili) et dans une certaine mesure le Brésil, le Mexique et le Venezuela avaient mis en place des formes d'Etat providence. Il est vrai que lorsqu'ils existaient, ces mécanismes redistributifs ont été liquidés par les militaires. Mais surtout, ils n'ont pas été remis sur place par les nouveaux gouvernements démocratiques, qui abandonnaient ainsi cet objectif. Inspirée directement par les organismes multilatéraux, la politique sociale n'a plus pour objectif d'assurer un semblant de cohésion sociale au niveau national, mais pour seul but de compenser les effets des politiques néolibérales en s'adressant, sur une base individuelle, à ceux qui sont sous le seuil de l'extrême pauvreté. Exemple parmi d'autres, le remplacement au Chili pendant la dictature militaire du système de retraites par répartition par un système par capitalisation. Le système de répartition chilien était relativement avancé pour la région, et couvrait une proportion significative de la population. Peu après le putsch de 1973, la junte militaire met fin à ce système, et le remplace par un système par capitalisation, expérience pionnière en la matière pour les économistes néo-libéraux. Or aucun des gouvernements élus de manière démocratique depuis 1989 n'est revenu sur ce système.

\section{L'alternative hors des institutions politiques}

Enfin, dernière conséquence, et non des moindres, de l'adoption de politiques néolibérales : l'abandon de fait de l'idée que le politique est le lieu de l'affrontement entre idées et modèles alternatifs. Aucun parti d'importance ne conteste dans un premier temps l'application du modèle néo-libéral. Et peu nombreux sont les dirigeants politiques à exiger que les cas de corruption et de concussions révélées lors des privatisations massives soient réellement et sévèrement punis, au-delà de quelques cas ponctuels. Les seules possibilités de débat semblent cantonnées à l'ampleur des politiques sociales qu'il convient de mettre en oeuvre pour compenser les effets des réformes. D'une certaine manière, le rétablissement relativement rapide des équilibres macro-économiques conduit à renforcer le consensus autour de ces politiques parmi les dirigeants.

Désenchantement, dégradation, inachèvement ${ }^{4}$, faible qualité de ces nouvelles démocraties, autant de constats qui rendent compte de cette situation paradoxale. Les règles démocratiques sont acceptées par tous. Elles survivent à une situation socio-économique dramatique. Mais ce changement institutionnel considérable n'a pas conduit à l'idée que des élections transparentes et une pluralité de partis, pouvaient signifier l'occasion d'un changement conséquent de politiques. Il n'est pas étonnant de ce fait que dans les années 1990, ce soient des acteurs hors des institutions politiques centrales qui aient d'une manière ou d'une autre incarné la possibilité d'une alternative ou d'un changement.

\section{L'émergence de nouveaux dirigeants}

Par-delà leurs différences, le soulèvement zapatiste au Mexique et les mouvements indiens en Equateur et en Bolivie ont cherché à combiner à la fois des revendications spécifiques aux droits des populations indiennes et la défense de mécanismes et de processus de représentation et délibération inspirés de la démocratie directe. Or à partir de ces questions, ces mouvements vont formuler une critique plus large aux modèles néo-libéraux, ce qui

\footnotetext{
${ }^{4}$ Alvaro ARTIGAS, Amérique du sud, les démocraties inachevées, Paris, Colin, 2005.
} 
contribue à contester l'acceptation qui semblait jusque là généralisée. De manière ponctuelle, ces actions parviennent à leurs buts : une mobilisation de la population de la ville de Cochabamba en Bolivie en 1999 et 2000 conduit ainsi à imposer de revenir sur la privatisation des services de distribution de l'eau'.

Autre exemple de l'affaiblissement de la scène politique centrale : dans de nombreux pays, la tendance à la fragmentation locale de la politique s'accentue, de nouveaux dirigeants émergent, pour la plupart nouveaux venus en politique, qui font de leur inexpérience un argument de campagne face à des électeurs désabusés. Cet éclatement constitue un symptôme de l'incapacité grandissante des scènes politiques centrales à articuler une forme de cohésion nationale. Mais le niveau local constitue également le lieu de tout un ensemble d'expériences concrètes qui présentent sinon des alternatives aux politiques gouvernementales du moins des processus de réinvestissement du politique par les citoyens, à l'instar de l'expérience du budget participatif de Porto Alegre ${ }^{6}$. Repris et réadapté ailleurs, le système conduit à réhabiliter l'idée de l'existence d'un espace public urbain, lieu à la fois de délibération et $\mathrm{d}^{\prime}$ action pour les citoyens et pour les pouvoirs locaux ${ }^{7}$. Enfin, phénomène inédit jusque là : des présidents, élus démocratiquement, doivent quitter le pouvoir non sous la pression des militaires mais soit à la suite de procédures d'impeachment, soit sous celle de manifestations massives, par lesquelles la population proteste à la fois contre les cas de corruption, de fraude et contre les excès des politiques néo-libérales. En 1992 le Président brésilien Collor de Mello est démis de ses fonctions par le Parlement pour corruption. Les cas postérieurs tiennent davantage de pratiques extra-institutionnelles. C'est le cas, déjà évoqué, d'Alberto Fujimori au Pérou en 2000. C'est également le cas lors de la crise argentine de 2001-2002. Après plusieurs années d'une croissance maintenue par l'existence d'un cours forcé de la monnaie nationale à parité avec le dollar, la situation économique s'est dégradée. Le pays a connu une très sérieuse récession, menant à de nombreuses faillites d'entreprises mais également d'administrations publiques, incapables d'honorer les salaires de leurs employés. En décembre 2001, suite à des manifestations massives qui demandent le départ de tous les dirigeants politiques, le président $\mathrm{De}$ La Rua démissionne. Le nouveau gouvernement abandonne la politique monétaire de parité forcée. Des scénarios identiques se reproduisent en Equateur en 2000 puis en 2005, ainsi qu'en Bolivie en 2003 et 2005 ; dans ce pays, les manifestants réclament en même temps le départ des présidents en place et une renationalisation des ressources gazières du pays.

\section{Le retour du rôle de l'Etat}

Le lien entre ces mouvements sociaux et les partis politiques, en particulier de gauche, est variable selon les pays : absent en Argentine, il évolue au Mexique entre formes d'appui et tensions très vives, alors qu' en Equateur et surtout en Bolivie, le parti politique est avant tout pensé comme un instrument des mouvements sociaux pour être présent dans les institutions et obtenir des postes de représentation. Mais dans leur diversité, ces mobilisations concourent à intégrer des formes de critique aux modèles néo-libéraux au sein de la sphère partisane et obligent d'une certaine manière les partis politiques à reprendre à leur compte certaines de leurs revendications. Ils rejoignent en ce sens le discours de certains dirigeants politiques élus, à l'instar de Hugo Chávez, président du Venezuela depuis 1999, qui s'est imposé

\footnotetext{
${ }^{5}$ Franck POUPEAU, « La guerre de l'eau, Cochabamba, Bolivie, 1999-2001 », Revue Agone, 26-27, 2002.

${ }^{6}$ Il s'agit d'attribuer le choix d'une part des investissements municipaux d'infrastructure à un conseil des habitants, constitué de représentants de conseils locaux où chacun peut présenter et défendre les choix d'investissement décidés collectivement. Voir Marion GRET, Yves SINTOMER, Porto Alegre, l'espoir d'une autre démocratie, Paris, La Découverte, 2002.

${ }^{7}$ Olivier DABENE, Exclusion et politique à Saõ Paulo, Paris, Karthala, 2006.
} 
régionalement comme la figure d'une position critique au modèle néo-libéral. Sans être aussi radicaux, nombre de partis de gauche entendent à nouveau incarner une alternative aux gouvernements en place.

Or cette différence se fait entendre, y compris au sein des formations les plus modérées, justement sur l'une des thématiques mise en avant par les mouvements sociaux, la réhabilitation du rôle de l'Etat dans son rôle de garant d'une forme de cohésion sociale. En 2002 au Brésil, avec la victoire de Luis Inacio Lula da Silva à la Présidence, en 2003 en Argentine (Néstor Kirchner) et en 2005 en Uruguay (Tabaré Vázquez), des partis ou candidats de la gauche modérée parviennent au pouvoir. Ce qui les différencie de leurs adversaires politiques est la volonté explicite de réhabiliter la politique sociale comme mécanisme de redistribution au bénéfice des plus pauvres, au nom d'une certaine conception de la cohésion nationale. Au Chili, c'est sur cette question que la socialiste Michelle Bachelet, élue en 2006, a voulu affirmer sa différence, alors même qu'elle succède à un président lui-même socialiste et qu'elle est présentée par la même coalition de partis de centre et de gauche qui gouverne le pays sans discontinuité depuis 1990, sans remise en cause majeure des options néo-libérales du régime militaire en matière économique et sociale. C'est sur cette question que la gauche mexicaine a fait campagne lors des élections présidentielles de 2006, après avoir mis en œuvre avec un certain succès des programmes de redistribution destinés aux personnes âgés et plus vulnérables dans la ville de Mexico. Et enfin, c'est sur cette thématique en particulier que les partis ou candidats de la gauche dite radicale souhaitent incarner un changement. Les victoires électorales en 2006 d'Evo Morales en Bolivie, de Rafael Correa en Equateur, de Daniel Ortega au Nicaragua doivent se comprendre au regard de cette volonté.

Il va de soit que la marge d'action de l'ensemble de ces gouvernements de gauche ou de centre-gauche élus récemment en Amérique latine est particulièrement limitée et que cela pèse sur leur capacité à mettre en œuvre effectivement des politiques de redistribution. Seul le président vénézuélien dispose des ressources suffisantes pour financer sur le long terme une politique sociale. Néanmoins, les mobilisations sociales dans un premier temps, puis plus récemment les succès électoraux de la gauche ont contribué à réinvestir le politique de deux des éléments balayés par la crise économique et le modèle néo-libéral : d'une part, les scènes électorales sont à nouveau le lieu de l'expression d'un clivage entre options programmatiques différentes; d'autre part, cette alternative se cristallise justement sur le rôle de l'Etat en matière de redistribution. Pour certains l'Etat doit être un acteur central de la redistribution sociale, pour d'autres au contraire, il n'a pour seul rôle que d'assurer le libre jeu des règles du marché. C'est par ce double réinvestissement que les institutions démocratiques en Amérique latine peuvent être effectivement un lieu de la discussion et de la mise en oeuvre du changement social. 